\title{
Low frequency somatic copy number alterations in normal human lymphocytes revealed by large scale single-cell whole genome profiling
}

Lu Liu ${ }^{1, \#}$, He Chen ${ }^{1, \#}$, Cheng Sun ${ }^{2, \#}$, Jianyun Zhang ${ }^{3, \#}$, Juncheng Wang ${ }^{2}$, Meijie Du ${ }^{4}$, Jie $\mathrm{Li}^{4}$, Lin $\mathrm{Di}^{1}$, Jie Shen ${ }^{5}$, Shuang Geng ${ }^{1}$, Yuhong Pang ${ }^{1}$, Yingying Luo ${ }^{6}$, Chen $\mathrm{Wu}^{6}$, Yusi $\mathrm{Fu}^{1, *}$, Zhe Zheng, ${ }^{2, *}$, Jianbin Wang ${ }^{4, *}$, and Yanyi Huang ${ }^{1,7,8,9, *}$

${ }^{1}$ Biomedical Pioneering Innovation Center (BIOPIC) and Beijing Advanced Innovation Center for Genomics (ICG), School of Life Sciences, Peking University,

11 Beijing 100871, China.

$12{ }^{2}$ National Clinical Research Center of Cardiovascular Diseases, State Key Laboratory 13 of Cardiovascular Disease, Fuwai Hospital, National Center for Cardiovascular

14 Diseases, Chinese Academy of Medical Sciences and Peking Union Medical College, 15 Beijing 102300, China.

$16{ }^{3}$ Department of Oral Pathology, Peking University School and Hospital of

17 Stomatology, National Engineering Laboratory for Digital and Material Technology

18 of Stomatology, and Beijing Key Laboratory of Digital Stomatology, Beijing 100081, 19 China.

$20{ }^{4}$ School of Life Sciences and Beijing Advanced Innovation Center for Structural

21 Biology (ICSB), Tsinghua University, Beijing 100084, China.

$22{ }^{5}$ Department of Neurobiology, Capital Medical University, Beijing100069, China.

$23{ }^{6}$ Department of Etiology and Carcinogenesis, National Cancer Center/Cancer

24 Hospital, Chinese Academy of Medical Sciences and Peking Union Medical College, 25 Beijing 100021, China.

$26{ }^{7}$ College of Chemistry and Molecular Engineering, Beijing National Laboratory for

27 Molecular Sciences, Peking University, Beijing 100871, China.

$28{ }^{8}$ Peking-Tsinghua Center for Life Sciences, Peking University, Beijing 100871,

29 China.

$30{ }^{9}$ Institute for Cell Analysis, Shenzhen Bay Laboratory, Shenzhen 518132, China.

$31 *$ Correspondence to: fuyusi.does@gmail.com (Y.F.), zhengzhe@fuwai.com (Z.Z.),

32 jianbinwang@tsinghua.edu.cn (J.W.), yanyi@pku.edu.cn (Y.H.).

33 \# These authors contributed equally to this work. 


\section{Abstract}

Genomic-scale somatic copy number alterations in healthy humans are difficult to investigate because of low occurrence rates and the structural variations' stochastic natures. Using a Tn5-transposase assisted single-cell whole genome sequencing method, we sequenced over 20,000 single lymphocytes from 16 individuals. Then, with the scale increased to a few thousand single cells per individual, we found that about $7.5 \%$ of the cells had large-size copy number alterations. Trisomy 21 was the most prevalent aneuploid event among all autosomal copy number alterations, while monosomy $\mathrm{X}$ occurred most frequently in over-30-year-old females. In the monosomy $\mathrm{X}$ single cells from individuals with phased genomes and identified Xinactivation ratios in bulk, the inactive $\mathrm{X}$ Chromosomes were lost more often than were the active ones.

\section{Introduction}

Genomic alterations, including copy number alterations (CNAs) and point mutations, are the major drivers of many cellular malfunctions (Conrad et al. 2010; Sudmant et al. 2015). Tumor cells, for example, usually carry many CNAs and point mutations

52 (Beroukhim et al. 2010; Waddell et al. 2015), many of which are oncogenic. After

53 decades of study, researchers now recognize that point mutations accumulate in 54 normal cells through polymerase replication errors and damaged DNA. Many point mutations barely affect cells, while others, located at critical locations, can transform

56 cells (The Wellcome Trust Case Control Consortium 2010; Klopocki et al. 2011). The

57 scenario for CNAs in normal cells is less clear. While large CNAs are extremely rare

58 in humans, thus suggesting their destructive potential in cells (Zhang et al. 2009;

59 Girirajan et al. 2011; Zarrei et al. 2015), their occurrences in normal cells may have

60 been underestimated due to technical constraints.

61 Recent advances in single-cell sequencing have greatly extended our understanding of 
cellular complexity (Vitak et al. 2017; Zahn et al. 2017; Chen et al. 2017). However, few studies have reported the heterogeneities of nuclear genomic variations in single cells (Rohrback et al. 2018; Laks et al. 2019). Various technologies have estimated that the frequencies of somatic CNAs in the human brain vary between $2 \%$ and $40 \%$ (McConnell et al. 2013; Cai et al. 2014; Knouse et al. 2014; van den Bos et al. 2016; Chronister et al. 2019). The current lack of a scalable and robust method to perform uniform single-cell whole genome amplification (WGA) is the main challenge to improving the accuracy and precision of somatic CNA identification.

In this report, we present a high-throughput single-cell WGA and sequencing method:

71 Tn5-transposase-assisted single-cell whole genome sequencing (Tasc-WGS). We

72 demonstrate the power of Tasc-WGS by showing the results of a large scale

73 investigation that identified rare CNA events in the lymphocytes from 16 cancer-free

74 individuals of different ages and sexes. We portrayed the CNA pattern of normal

75 lymphocytes and discovered hot spot regions. Combined with haplotype and

76 transcriptome data, we were able to reveal the biological bias of aneuploid events in

77 Chromosome X.

\section{Results}

79 A high-throughput pipeline for single-cell somatic CNA analysis

80 We optimized our Tasc-WGS method by forgoing both pre-amplification and library

81 quantification, and by directly tagmenting the double-stranded genomic DNA of

82 single cells, thus greatly simplifying the experimental process and increasing

83 throughput (Fig. 1A). Previous studies had shown that the library construction

84 protocol performed in a microfluidics system enables uniform amplification of a

85 single-cell genome (Zahn et al. 2017; Laks et al. 2019). We expanded that protocol to 86 96-well plates, and our subsequent performance evaluation verified that our protocol

87 provided even amplification and little contaminated data, as well as a marked increase 

in throughput that needed no special instrumentation (Supplementary Fig. S1A, B, C). We typically processed about 2,000 single cells in a single experimental run and used cellular barcodes to differentiate each cell's sequence reads (Table S2).

With a shallow sequencing depth $(\sim 0.1 \times$; i.e., $0.3 \mathrm{~Gb}$ per cell $)$, we obtained an average $3.50 \% \pm 1.50 \%(\mathrm{CI}=95 \%)$ genome coverage and detailed copy number profile with $200-\mathrm{kb}$ bins. The coverage was uniform across the whole genome (Supplementary Fig. S1A), with no crosstalk between samples (Supplementary Fig. S1B, Supplementary Notes). We combined circular binary segmentation and hidden Markov model algorithms to further reduce false identification of unambiguous copy number losses or gains (Supplementary Fig. S1D, E, Supplementary Notes). Most of the quality filtering of single cell sequencing data was done with combinatorial criteria, including mean absolute deviation of pairwise difference, degree of ploidy abnormality, and degree of fragmentation (Supplementary Fig. S1F, G,

\section{Supplementary Notes).}

Using a primary tumor sample cell-line and split-cell genome DNA as controls (Supplementary Fig. S2), we further validated the robustness of our Tasc-WGS protocol. First, we generated 96 libraries of 6-pg bulk, dilute, blood gDNA and then used it to show that tagmentation reactions and PCR occurred uniformly across the whole genome (Supplementary Fig. S2B, C). To evaluate the sensitivity of TascWGS, we sequenced both a bulk and 29 single-cell samples of the HEK293 cell line, subsequently finding our method capable of detecting various types of CNAs ranging in size from $1 \mathrm{Mb}$ to $26 \mathrm{Mb}$ : $\mathrm{CN}$ gains $\geq 3.0$, range 3.06-7.91, median =3.60; copy number losses $\leq 1.3$, range 1.20-1.30, median $=1.28$ (Supplementary Fig. S2D, E). To evaluate the specificity of Tasc-WGS, we conducted CNA calling on a GM12878 B-lymphoblastoid cell line. First, bulk DNA genome profiling of GM12878 proved the diploid karyotype, and then we found some subpopulations with shared CNAs, as well as some unique CNAs, in the 49 GM12878 single-cell sequences 
115 (Supplementary Fig. S2F). Most of those unique CNAs ranged in size from $0.6 \mathrm{Mb}$

116 to $10 \mathrm{Mb}$ (Supplementary Fig. S2G, Table S4). So, to examine whether those small

117 CNAs could have been data point noise mistakenly called by the algorithm, we

118 simulated normally distributed copy number profiles that had multiple noise levels

119 (Nilsen et al. 2012, Supplementary Fig. S3). As expected, noisier data tended to have

120 higher false positive (FP) rates and FP CNAs were all small, ranging from $0.6 \mathrm{Mb}$ to

$1214.8 \mathrm{Mb}($ mean $=2.3 \mathrm{Mb})($ Table S5). Although it is possible that small CNAs $(<10$

$122 \mathrm{Mb}$ ) occurred more because they affected fewer genes and thus affected cell survival

123 less than larger CNAs do, it is hard to distinguish between true- and false-positive

124 calls of small CNAs. Therefore, for the lymphocyte samples, we decided to include

125 only CNA calls larger than $2 \mathrm{Mb}$.

\section{Somatic CNA events occurred commonly in lymphocytes}

127 We examined 33,600 single lymphocytes sorted from the blood of 16 cancer-free

128 individuals (10 males and 6 females; $1,440-3,840$ single lymphocytes per individual)

129 aged 9 months to 80 years (Fig. 1B, Table S1). Among them, 31,125 cells (92.6\%)

130 had more than 0.3 million reads aligned to the reference genome, and 20,594 cells

131 (61.3\%) passed the aforementioned quality filtering criteria for CNA analyses (Fig.

132 1C, Supplementary Fig. S4A-E, Supplementary Notes).

133 We found somatic CNA-containing lymphocytes in all individuals and identified 1344,809 cells ( $24.0 \%$ on average, $12.9 \%-44.5 \%$ for different individuals) harboring 135 small CNAs (2-10 Mb) and 1,500 cells (7.3\% on average, 3.3\%-15.2\% for different 136 individuals) harboring large CNAs ( $>10 \mathrm{Mb}$ ) (Fig. 1D, Supplementary Fig. S4F). 137 Furthermore, we observed a few cells, from different individuals, that carried similar 138 CNAs. Some other cells carried multiple CNAs across the whole genome (Fig. 1D). 139 As in previous reports (Knouse et al. 2016), copy number deletions occurred much 140 more often than did copy number amplifications (Supplementary Fig. S4G, H), and 
141 the ratios of CNA-containing cells were similar between males and females

142 (Supplementary Fig. S4I). Because large CNAs may affect more genes and cause

143 more serious problems than small CNAs, we focused on the large somatic CNAs

144 (excluding Chromosome Y due to technical challenges) that we had identified in

1451,397 lymphocytes.

146 We examined a large number of cells to minimize sampling error and to accurately

147 assess the ratio of CNA-containing lymphocytes. To that end, we ran a simulation to

148 determine the optimal number of cells that had to be sequenced to accurately assess

149 the ratio of CNA-containing cells, and found that a smaller sample invariably yields

150 uncertain assessment results (Fig. 1E). A throughput of about 1,000 or more cells per

151 sample was ideal to achieve a coefficient of variation (CV) below 20\%, and thus

152 accurate CNA assessment with occurrence ratios less than $10 \%$. We then checked

153 whether those somatic, megabase-size CNAs in lymphocytes were age related

154 (Machiela et al. 2015; Vattathil et al. 2016), and found a relatively weak correlation

155 (Fig. 1F). All our observations suggested that CNAs were common in lymphocytes of 156 cancer-free individuals.

\section{Large-size autosomal somatic CNAs occurred randomly in lymphocytes}

158 We further analyzed CNA profile similarities between cells to try to capture clonal

159 amplification signatures. Dimension-reduction analysis of single-cell copy number

160 profiles produced a few clusters based on large-CNA patterns (Fig. 2A,

161 Supplementary Fig. S5A, Supplementary Notes). Chromosome 21 and Chr X

162 CNAs separated from the others, mostly because of their distinct aneuploidy patterns,

163 but we observed no other obvious clustering based on sex, sample, or chromosome

164 (Supplementary Fig. S5A). Using pairwise Euclidean distances of CNA profiles, we

165 found 51 cell clusters, all with potential clonal cell amplifications (Fig. 2B-D,

166 Supplementary Fig. S5B, Supplementary Notes). Although clonal amplifications 
167 among lymphocytes are not common (cell ratio $=2.3 \%$, median clone size $=4$ ), we

168 found that most individuals (8/10 males and 6/6 females) exhibited such events, with

169 clone sizes ranging from 3 to 105 cells. Aside from the characteristic aneuploid clones

170 of Chr 21- and Chr X-containing cells, the largest clone, in F01's Chromosome 6,

171 contained 11 cells with an approximate $35 \mathrm{Mb}$ loss (Fig. 2E).

172 Somatic CNA events were scattered across every chromosome (Fig. 2F), although all

173 CNAs together could cover almost the entire genome (99.4\%). For each genomic

174 locus that contained CNA events, the occurrence frequency was less than $1 \%(0.0 \%-$

175 1.0\%), except for Chr X (1.3\%-1.8\%) (Supplementary Fig. S5C). We detected no

176 shared CNA hotspots among the participants, and CNA distributions were no different

177 than a random distribution (Fig. 2G), except for the obviously higher frequencies at

178 Chrs 21 and X. In keeping with the random generation mechanism, longer

179 chromosomes contained proportionally more CNAs than shorter ones did (in a linear

180 relationship, Fig. 3A), while Chr 21 and Chr X exhibited significantly higher

181 occurrence rates than other chromosomes. We then examined whether certain

182 chromosomes were more prone to CNAs than others and found that, except for Chr X

183 and Chr 21, all other autosomes showed similar CNA count densities (Fig. 3B,

184 Supplementary Fig. S5D).

185 CNA size distribution showed that copy number amplifications affect larger CNAs

186 more than do deletions and they were usually aneuploidies (Supplementary Fig.

187 S6A). Specifically, excepting Chr 21, 57.6\% (38/66) of the autosomal copy number

188 amplifications were aneuploidies, while only $2.0 \%$ of the deletions were $(22 / 1123)$

189 (Supplementary Fig. S6B, C). These results suggest that the mechanism for copy

190 number gain may be different than that for loss.

\section{Aneuploidy occurred mainly at Chr 21 and the sex chromosomes}

192 We identified somatic aneuploidy in almost all chromosomes, but with a $2.4 \%(n=$ 
193 498) cellular occurrence rate, it can be easily missed if the experimental throughput is

194 not large enough. We verified low-frequency autosomal aneuploidy using

195 fluorescence in situ hybridization assays, and quantitatively confirmed the copy

196 number gains and losses in Chr 3, 8, 13, 18, 21 and X (Supplementary Fig. S5E,

197 Table S3).

198 Among all the aneuploid cells, aneuploidy in Chr X predominated (35 gains and 235

199 losses, 52.5\% of the total events), followed by Chr 21 (48 gains and 21 losses, 13.4\%

200 of the total events) (Supplementary Fig. S6D). The remaining 60 cells contained

$201 \quad 11.6 \%$ of the total aneuploidy events (38 gains and 22 losses).

202 Chromosome 21 had about half of the autosomal aneuploidy events (Supplementary

203 Fig. S6E), with more gains than losses. We noticed that Chr 21 aneuploidy occurred

204 unevenly among individuals (Supplementary Fig. S6F), as M10 showed a

205 significantly higher ratio of trisomy $21(2.5 \%$ versus $0.2 \%)$ than other individuals did.

206 Among all individuals, trisomy 21 occurred more in males than in females

207 (Supplementary Fig. S6F), but monosomy 21 occurred equally between males and 208 females (Supplementary Fig. S6G).

209 The aneuploidy occurrence rate for Chr X (270 cells, $1.31 \%$ in total) was significantly 210 higher than that of the other chromosomes, contributing to $72.2 \%$ of the cells with

211 CNAs $>10$ Mb (374 cells) (Supplementary Fig. S6H). Although all male Chr X

212 aneuploid cells $(\mathrm{n}=15)$ were disomic, the majority of $\mathrm{Chr} 21$ aneuploidy events were

213 monosomic (235 cells, 87.0\%) and occurred mostly in females ( 255 female cells, 15

214 male cells, Supplementary Fig. S6H). Such Chr X loss is prevalent in females

215 aged $>30$ years ( 228 cells), but is rarely discovered in young females ( 7 cells).

216 Identifying aneuploidy events in Chr Y was challenging because of its short unique

217 genomic regions ( $\sim 17 \mathrm{Mb})$. Therefore, we relied on reads counts, instead of copy

218 number estimations, to deduce Chr Y ploidy number, subsequently identifying 115 
male cells with Chr Y loss ( $0.9 \%$ of all male cells). To verify the reliability of this

220 assessment, we used reads counts to analyze $\mathrm{Chr} \mathrm{X}$ and $\mathrm{Chr} 21$ aneuploidy and

221 compared those results with the previous ones (Fig. 3C-F). The reads count

222 distribution had a clear linear relationship among monosomic, normal, and trisomic

223 cells, and almost all the results were consistent with the bin-based method (98.37\%).

\section{Loss of heterozygosity and allelic bias in copy number alterations}

225 Using phased, personalized genomic information from F03 and F06 (Fig. 4A,

226 Supplementary Notes), we investigated whether somatic CNA events were allele

227 specific. We analyzed all copy neutral chromosomes $(\mathrm{CN}=2)$ in 2,668 cells from F03

228 and F06 and found only 4 cells ( 2 from F03 and 2 from F06, 0.1\% and $0.2 \%$ per

229 individual, respectively) contained copy neutral loss of heterozygosity events in

230 Chromosomes 1, 14, 21, and 22 (Fig. 4B). We then focused on $\mathrm{CN}=1$ genomic

231 regions, within which the allelic pattern should have been either single parental or

232 segmental. Of the $72 \mathrm{CN}=1$ events that we analyzed, $70(97.2 \%)$ were single

233 parental and the other two had shuffled genotypes without segmental patterns (Fig.

234 4C-E, Supplementary Fig. S7), likely due to the low probability of collision

235 between a CNA occurrence and recombination.

236 We next examined whether monosomy X cells were parentally biased during

237 chromosome loss, and found most of the F03 monosomic X lymphocytes $(86.2 \%$,

$23825 / 29$ cells) were maternal, while most of the affected F06 monosomic X

239 lymphocytes were paternal (86.0\%, 37/43 cells) (Fig. 4C-E, Supplementary Fig.

240 S7). To exclude possible technical artifacts and contamination, we calculated the

241 single-nucleotide polymorphism (SNP) density ratio between Chromosome X and

242 Chromosome 10 and found that the ratio distributions for both normal and monosomy

243 X cells were similar (Fig. 4F). However, when calculating the normalized reads

244 numbers, we found that the distribution of reads ratio between $\mathrm{Chr} \mathrm{X}$ and 10 for 
245 normal cells was similar, but it was inconsistent for monosomic cells, as only half the

246 reads mapped to $\mathrm{Chr} \mathrm{X}$ (Fig. 4G).

247 To examine whether parental preference in Chr X loss might correlate with Chr X

248 inactivation, we conducted bulk RNA-seq, based on phased SNPs, to determine F03's

249 allele-specific expression across her whole genome (Supplementary Notes). As

250 expected, autosomal genes expressed unbiased biallelic expression (Fig. 4H,

251 Supplementary Fig. S7), but Chr X expression was greatly biased toward the

252 maternal allele, coincident with the fact that the Chr X loss in F03 was mainly

253 paternal (Fig. 4C, D).

\section{Discussion}

255 In this study, we used high-throughput Tasc-WGS to profile CNA landscapes of more 256 than 20,000 single lymphocytes sampled from 16 individuals. Even though CNAs 257 were detected in blood lymphocytes of every donor, the occurrence rate of CNAs $>10$

$258 \mathrm{Mb}$ for each individual was rather low and correlated little to age. Such low-

259 frequency events can be accurately identified only by profiling thousands of single 260 cells per person; and from the technological perspective, the scalability of single-cell

261 WGS is key to enabling such observations. Additionally, a benefit of large-scale high262 performance single-cell sequencing libraries is that we can use them to identify rare 263 CNA events at high resolution, a formerly impractical achievement using other 264 throughput-limited single-cell WGA methods, or by using bulk samples.

265 We found that CNAs, including the large ones $(>10 \mathrm{Mb})$, were widely distributed 266 throughout the lymphocyte genomes; thus revealing that, on average, about $5 \%$ of the 267 lymphocytes of a healthy human have large CNAs. Also, losses were more prevalent 268 than gains, thus suggesting that losses occur more readily than gains do. Previous 269 studies of neurons showed a similarly scattered CNA pattern throughout the genome 270 (Cai et al. 2014; Chronister et al. 2019). Some researchers think that the activity of 
271 mobile elements, like that of the active long interspersed nuclear element 1 during

272 brain development, is a major cause of CNAs (Evrony et al. 2015; Sanchez-Luque et

273 al. 2019; Richardson et al. 2014; Erwin et al. 2016). However, unlike that of neurons,

274 the lymphocyte regeneration rate is high, and CNAs harbored in precursor cells may

275 pass to descendent cells via cell differentiation or division. Indeed, most CNA-

276 containing cells are not clonal—we observed only 51 clones in our 20,594-cell

277 sample. In terms of the number of cells, the sizes of these clones were insignificant,

278 thus indicating that rather than being generated in the early developmental stages,

279 they were newly generated. Lymphocytes can expand through mitosis, so some low-

280 frequency CNA events may be inherited.

281 The CNAs seemed to occur in no particular location across the genome, except in

282 Chromosomes 21, X, and Y, where most of the aneuploidies were found. Most

283 segmental CNAs were randomly scattered across the whole genome, but whole-

284 chromosome CNAs likely affected many genes, either by completely silencing or

285 altering their expression levels (Zhang et al. 2009; Girirajan et al. 2011). As a result,

286 aneuploidies are lethal in most cases (Hassold et al. 2001; Santaguida et al. 2015).

287 However, $2.4 \%$ of the cells in our study had aneuploid events, especially at Chr 21 ,

288 which displayed trisomy, the most prevalent human aneuploidy (Sanchez-Luque et al.

289 2019; Richardson et al. 2014). This consistency suggests similar selection outcomes

290 for aneuploid events in both humans and lymphocytes.

291 Aneuploidy occurs more in sex chromosomes than in autosomes. Although our results

292 showed a weak correlation of age with CNA occurrence, monosomy X is rare in

293 young females but becomes more prevalent in females over age 30, for whom the rate

294 can reach $2 \%-7 \%$. Such a chromosome loss was parentally allele specific in the

295 affected individuals. According to haplotype information and bulk RNA-seq data, we

296 found that most lost X Chromosomes were inactive (Xi). These results agree with

297 previous studies that suggested that the $\mathrm{X}$ inactivation skewness pattern is more 
298 prevalent in older than in younger individuals (Sharp et al. 2000; Sandovici et al.

299 2004; Amos-Landgraf et al. 2006; Machiela et al. 2016; Zito et al. 2019). This

300 association may be due to the lethality of a lost active $\mathrm{X}$, so most monosomy $\mathrm{X}$ cells

301 are missing Xi because negative selection assumes a randomly generated loss during

302 mitosis. Unlike the high incidence of monosomy X cells, trisomy X cells are rarely

303 found. This imbalance further suggests that Chromosome $\mathrm{X}$ aneuploidy likely does

304 not result from simple uneven separation during mitosis. Additionally, compared with

305 autosomal aneuploidies, $\mathrm{Xi}$ loss is both non-lethal to affected cells and under positive

306 selection. Therefore, we observed a higher incidence of monosomy X cells than cells

307 with autosomal events. Another possible contributing factor is that conformational

308 changes to Xi may ease its loss during mitosis (Galupa et al. 2018). Obviously, more

309 investigation is needed to clarify the mechanisms of these phenomena.

310 Our results demonstrate how shallow WGS, after extending throughput to 1,000

311 single cells, enables quantitative identification of rare copy number change events.

312 However, the sensitivity of CNA detection was limited in this study because, although

313 it performs better than most other existing methods, Tasc-WGS harbors intrinsic

314 coverage noise due to amplification bias and coverage stochasticity. To lower that

315 false positive CNA identification rate, we applied strict criteria to screen such events,

316 even though it caused us to lose some sensitivity and resolution. Studies of cancers

317 (Beroukhim et al. 2010; Navin et al. 2011; Jacobs et al. 2012; Laurie et al. 2012) and

318 neuronal disorders (van den Bos et al. 2016; Yurov et al. 2007; Bundo et al. 2014)

319 would benefit from the ability to identify smaller CNAs, but improvements of both

320 experimental protocols and computational algorithms are needed. For instance, the

321 commonly used data processing pipelines for determining copy number are based on

322 bulk sequencing or micro-array data, not single-cell data. With the popularity of

323 single-cell profiling and the availability of more data, such as ours, a need for more

324 appropriate computational approaches must be met soon. 
327 Ethics approval

328 This study was approved by the Ethics Committee of Tsinghua University (No.

329 20180011), Ethics Committee of the Cancer Hospital, the Chinese Academy of

330 Medical Sciences and Peking Union Medical College (No. NCC2017G-002), and the

331 Ethics Committee of Fuwai Hospital, Chinese Academy of Medical Sciences and

332 Peking Union Medical College (No. 2017-880).

$333 \quad$ Patients and clinical samples

334 We recruited Fuwai Hospital patients who had cardiovascular diseases, as well as

335 their visiting family members. A single patient with colon cancer was enrolled

336 through the Cancer Hospital. Patients and family members were given full research

337 program descriptions, which included potential risks. We obtained informed consent

338 from all patients and family members before genetic testing, and then collected fresh

339 blood samples from both healthy donors (M01-M03, F03-F06) and cardiovascular

340 disease patients (M04-M10, F01, F02), as well as a tumor sample from the cancer

341 patient after surgery.

$342 \quad$ Peripheral blood mononuclear cell isolation and single-cell sorting

343 We used Ficoll-Paque PLUS (Cytiva, \#17-1440-02) according to the manufacturer's

344 instructions to isolate mononuclear cells from fresh blood samples. Briefly, for each

345 sample, Ficoll-Paque medium ( $3 \mathrm{ml})$ was added to a $15-\mathrm{mL}$ centrifuge tube and then a

346 blood sample $(2 \mathrm{~mL})$ diluted 1:1 in phosphate buffered saline (PBS) was carefully

347 layered onto the Ficoll-Paque medium. The tube was then centrifuged at $400 \mathrm{~g}$ for 30

$348 \mathrm{~min}$ at room temperature. The second layer, which contained mononuclear cells, was 
349 pipetted out, transferred to a new tube, and washed twice in $10 \mathrm{~mL}$ PBS before being

350 resuspended in $1 \mathrm{~mL}$ PBS- bovine serum albumin (BSA) buffer. Typically, we isolated

$3511 \times 10^{6}$ cells from each sample. We then used a FACSAria III sorter (BD

352 Biosciences), gated for lymphocytes and singlets, to sort out single cells according to

353 forward and side scatter signals. We then placed each sorted, single cell directly into 2

$354 \mu$ lysis buffer $(30 \mathrm{mM}$ Tris- $\mathrm{HCl}[\mathrm{pH}=8.0], 10 \mathrm{mM} \mathrm{NaCl}, 0.2 \mu \mathrm{L}$ Proteinase $\mathrm{K}$

355 [Qiagen, \#19133], 5 mM EDTA, and 0.5\% Triton X-100 [Sigma-Aldrich, \#T9284]) in

356 a well of a 96-well plate.

$357 \quad$ Single-cell isolation from a tumor tissue

358 We ground the colorectal cancer sample $\left(\sim 0.1 \mathrm{~cm}^{3}\right)$ using a dounce glass tissue

359 grinder. The cells were then washed, resuspended in PBS, and filtered through a

360 Falcon 40- $\mu \mathrm{m}$ cell strainer. They then underwent fluorescence-activated cell sorting

361 (FACS), gated for single-cells, and each cell was sorted into a well in a 96-well plate.

362 Culturing and isolating single cells and optimizing cell lines

363 We used GM12878 cells (Coriell Institute) and HEK293 cells (American Type

364 Culture Collection) for protocol optimization. Those cells were cultured at $37 \mathrm{C}^{\circ}$

365 under $5 \% \mathrm{CO}_{2}$ in a humidified incubator. We cultured GM12878 cells in RPMI 1640

366 medium (Gibco, \#C11875500BT) with 10\% fetal bovine serum (Gibco, \#10100147)

367 and 1\% penicillin-streptomycin (Gibco, \#15140122), then spun the cell suspension at

$368500 \mathrm{~g}$ for $5 \mathrm{~min}$, discarded the supernatant, and washed the cell pellet twice using PBS

369 before resuspending it in PBS with 1\% BSA. We cultured HEK293 cells in DMEM

370 medium (Gibco, \#11965092) with 10\% fetal bovine serum and 1\% penicillin-

371 streptomycin. The cells were then washed twice using PBS, detached by adding $1 \mathrm{~mL}$

$3720.25 \%$ trypsin-EDTA (Gibco, \#25200056) to their culture dish, centrifuged at $500 \mathrm{~g}$ for

$3735 \mathrm{~min}$, and recovered in 1\% PBS-BSA buffer. All cells underwent FACS that was

374 gated for single-cells and each cell was subsequently sorted to a well in a 96-well 
375 plate.

$376 \quad$ Purification of genomic DNA

377 We purified genomic DNA (gDNA) using a Genomic DNA Purification Kit (Thermo

378 Fisher Scientific, \#K0512) according to the manufacturer's instructions. We then

379 quantified that DNA with a Qubit fluorometer system (Invitrogen) and diluted it to 6 $380 \mathrm{pg} / \mu \mathrm{L}$.

$381 \quad$ Single-cell whole genome amplification and sequencing

382 The 96-well plates were then centrifuged at 2,000 $\mathrm{g}$ for $1 \mathrm{~min}$ and a lysis reaction 383 proceeded at $50{ }^{\circ} \mathrm{C}$ for $3 \mathrm{~h}$. We added tagmentation buffer (1x TD buffer, $0.015 \mu \mathrm{L}$ 384 TTE Mix V50 [Vazyme, \#TD501], 0.625x protease inhibitor cocktail [Promega, $385 \# \mathrm{G} 6521]$, and $1 \mathrm{mM} \mathrm{MgCl} 2$ ) to reach a volume of $10 \mu \mathrm{L}$ per well and then incubated 386 the plates at $55^{\circ} \mathrm{C}$ for $1 \mathrm{~h}$. Tagmented DNA fragments were amplified by adding 12 $387 \mu \mathrm{L}$ PCR master mix composed of $11 \mu \mathrm{L}$ Q5 High-Fidelity 2x Master Mix (New 388 England Biolabs, \#M0492) and 0.5 $\mu \mathrm{L}$ each of $10 \mathrm{mM}$ Nextera i5 and i7 index 389 primers. PCR thermocycling conditions were $72{ }^{\circ} \mathrm{C}$ for $8 \mathrm{~min}, 98^{\circ} \mathrm{C}$ for $30 \mathrm{~s}, 24$ 390 cycles of $98{ }^{\circ} \mathrm{C}$ for $15 \mathrm{~s}$ each, $60{ }^{\circ} \mathrm{C}$ for $30 \mathrm{~s}$, and $72{ }^{\circ} \mathrm{C}$ for $90 \mathrm{~s}$, with a final 391 incubation at $72{ }^{\circ} \mathrm{C}$ for $5 \mathrm{~min}$. The subsequent PCR products were merged in groups 392 of 5 plates (480 single-cell wells) and then purified using 1x VAHTS DNA clean 393 beads (Vazyme, \#N411). Library quality control was conducted on a 5200 Fragment 394 Analyzer System (Agilent, \#M5310AA) to determine fragment distribution, and then 395 qualified libraries were quantified and sequenced on a HiSeq X Ten System (Illumina) 396 following the manufacturer's standard protocols.

397 Bioinformatic analyses 
399 Paired-end reads were aligned to the human reference genome (hg38) using nvBowtie

400 (https://github.com/NVlabs/nvbio), a graphics processing unit-accelerated version of

401 Bowtie 2 (Langmead et al. 2009). Then, each cell's mapped reads were demultiplexed 402 using perfectly matched cell barcodes. Typically, 0.3 million reads were sufficient for 403 copy number profiling at a $200-\mathrm{kb}$ resolution. Before downstream analysis, we

404 excluded cells with less than 0.3 million reads, keeping reads mapped with minimum 405 mapping quality scores of 20, and removed PCR duplicates using SAMtools (Li et al. 406 2009).

408 We applied two methods, HMMcopy (Shah et al. 2006) and DNAcopy (Olshen et al. 409 2004), to calculate the copy number profiles of each sample at the $200-\mathrm{kb}$ resolution, 410 with GC content and mappability normalized. Both algorithms are commonly used in 411 single cell studies, but they each give different identification results for small size 412 variations (Knouse et al. 2016). HMMcopy uses a hidden Markov model (HMM) to 413 determine copy number, while DNAcopy applies circular binary segmentation (CBS)

414 for analysis. We combined the two methods to further increase the specificity and 415 accuracy of CNA identification.

416 We used the Bayesian information criterion as a metric to evaluate model fitness with 417 different computational parameters in HMMcopy and DNAcopy calculations, using 418 the strictest parameters (alpha $=10^{-4}$ for DNAcopy and e $=0.9999$ for HMMcopy) 419 under the same fitness to enhance CNA calling specificity.

420 After segmentation, we used three features to assess the quality of the single cell 421 sequencing results, and then filtered out low-quality cells and incorrect segmentation 422 calls. First, we checked the average of all copy numbers identified in each bin (degree 423 of ploidy abnormality), and that value was greatly influenced by cell ploidy. Cells 424 with abnormal ploidy at the whole genome level (ploidy $>3$ ) were discarded. We then 
425 checked the median absolute pairwise difference (MAPD), typically used for

426 indicating amplification evenness, to rule out poorly amplified cells (MAPD > 0.6).

427 Finally, we checked each cell's number of segments. We noticed that some cells

428 exhibited acceptable MAPD values but had fragmented copy number profiles. This

429 could have been caused by incomplete lysis, contamination from other cells or cell

430 debris, or during the S-phase, as some studies have suggested (Laks et al. 2019; Chen

431 et al. 2017). Since cells with a CNA or a fragmented chromosome will have more

432 segments and slightly higher MAPD values than would normal cells, the other

433 genomic regions of those cells are still high quality. So, we then calculated the

434 number of segments (degree of fragmentation) and MAPD for each chromosome and

435 used the third highest values to represent each cell's value.

Identification of copy number alteration events

437 We identified each CNA by combining the two algorithms, CBS and HMM, and

438 keeping the double-positive counts as true events. Since both algorithms are sensitive 439 to the local contents of copy number profiles (Knouse et al. 2016; Zhang et al. 2015), 440 especially for losses, we developed a shuffling pipeline to improve the confidence of 441 identifying CNA events.

442 For each cell with CNAs, segments with amplification or deletion were shuffled 443 throughout the genome and were re-identified by CBS and HMM algorithms. We 444 identified CNA events with high confidence by repeating the shuffle process 20 times 445 and averaging the copy number values identified for a given shuffled segment. A loss 446 was defined as a segment with a copy number value less than 1.4. Only those CNAs 447 larger than $2 \mathrm{Mb}$ were kept for downstream analysis.

448 To avoid false identification affected by mapping uniqueness, we ruled out those 449 CNAs either located near centromeres (overlapping more than 40\%) or with disperse 450 copy number profiles (with larger deviations [mean or median $>0.4$ ]) between the 
451 copy number values of bin and segment, usually at the chromosome ends).

453 Since bins of CNA profiles are normally distributed (Nilsen et al. 2012), we generated 454 normally distributed simulated CNA data to investigate false positive (FP) calls 455 introduced by the algorithm. We set $\sigma$ values to range from 0.4 to 0.8 and then 456 generated 1,000 simulated CNA profiles for each $\sigma$, replicating the process three 457 times. We then adopted the same CNA calling pipelines and counted the FP CNA 458 events in each batch.

460 Large CNAs are rare events and vulnerable to sampling errors. Therefore, we

461 simulated the sampling process by sampling different numbers of cells (sample size, 462 from 3 to $10^{5}$ ) from $10^{6}$ cells having different ratios of CNA-containing cells (from $4630 \%$ to $20 \%$ ). We repeated each test 100 times to determine sufficient sample size and 464 then calculated the coefficient of variation (CV) for each condition.

466 We first used dimension reduction to view all the cells with $>10 \mathrm{Mb}$ of CNAs. First, 467 CNA profiles having 200-kb resolutions were smoothed using a 4-Mb window; and 468 then, using multidimensional scaling, they were transformed into a low-dimensional 469 representation. We adjusted the number of dimensions representing each chromosome 470 (each dimension represents $\sim 10-\mathrm{Mb}$ CNA profiles) and concatenated all 471 chromosomes. Then, we were able to visualize low-dimensional representations of

472 CNA profiles in 2-dimensions by using $t$-stochastic neighbor embedding.

473 We identified clonal CNAs by calculating the relative CNA ratio in every 474 chromosome for every individual. Specifically, for each individual, we calculated 
475 either the fraction or the number of CNAs in each chromosome to represent the

476 enrichment of CNAs in each, and then normalized those values based on chromosome

477 lengths.

478 To further investigate the clonal CNAs, we calculated the Euclidean distance between

479 cells for every individual and identified similar cell pairs according to their distance

480 distributions. We then constructed an undirected graph using cells as nodes and the

481 Euclidean distances as edges and identified clones as maximally connected subgraphs.

482 Haplotype calling

483 We first genotyped genome sequencing data on all loci of the whole genome with the 484 same pipeline. Reads were first trimmed and filtered using the following criteria. The 485 adaptors were removed according to the reverse complementary sequence of the 486 paired-end reads, and filtered reads were dynamically trimmed with a Phred cutoff of 487 20. The remaining reads were then mapped to the human reference genome using 488 Bowtie 2 (MapQ $\geqslant 40, \mathrm{XM}<4$ ), and whole genome genotypes were called using the 489 UnifiedGenotyper mode of GATK-3.5 (DePristo et al. 2011). We performed 490 heterozygosity analysis with a minor allele frequency cutoff between $30 \%-50 \%$ and 491 with 0\%-20\% homozygosity. Variant call format (VCF) files of three sample 492 genotypes were merged into one VCF file, and heterozygous loci of those three 493 samples were extracted into a locus file as a union for VCF scanning. Only those loci 494 from which either the mother is heterozygous and father homozygous or the father is 495 heterozygous and mother homozygous were used to phase the child's haplotype.

\section{Analysis of Chr X}

497 For each single cell from F03 and F06, reads with single-nucleotide polymorphisms 498 (SNPs) were identified using SAMtools (base quality > 30). Then, the haplotype for 499 each SNP was labeled as paternal, maternal, or neither (likely due to sequencing 
500 error) using the haplotype map. Haplotype counts for each bin were the sums of every

501 SNP site in that bin. For monosomy X cells, SNPs in every bin within segments that

502 identified a loss, were summarized and identified as paternal, maternal, or

503 undetermined (binomial test, $p<0.001$ ).

505 Since Chr Y had few uniquely mapped reads, we had to develop a special method to

506 determine its copy number. Reads coverage of Chr 21, X, and Y were calculated by

507 SAMtools normalized by sequencing depth, and then cells with $\mathrm{Chr} 21$ and Chr X

508 aneuploidies were identified by coverage depth. If we calculated the percentage of

509 sequenced reads belonging to $\mathrm{Chr} 21$ or Chr X of each single cell, we could also

510 easily identify cells with normal or altered copy numbers in those two chromosomes.

511 Actually, we found that more than $98 \%$ of those results were consistent with the

512 results determined by coverage depth. We then applied our percentage classification

513 method to identify the Chr Y copy number for each single cell.

516 The whole-genome and RNA-seq data generated in this study are deposited in the

517 Genome Sequence Archive (GSA; https://ngdc.cncb.ac.cn/gsa-human) in National

518 Genomics Data Center, China National Center for Bioinformation / Beijing Institute

519 of Genomics, Chinese Academy of Sciences under accession number HRA001513.

520 The scripts generated for the bioinformatics analysis are available in the Supplemental

521 Code.

\section{Acknowledgements}

524 We thank Chenyang Geng from the Peking University High-throughput Sequencing 
525 Center and Biomedical Pioneering Innovation Center, and Fei Wang from the

526 National Center for Protein Sciences (Peking University) for the experimental

527 assistance. This project was supported by National Key R\&D Program of China

528 (2018YFA0108100 to Y.H.), National Natural Science Foundation of China

529 (22050002 to Y.H.), Beijing Municipal Science and Technology Commission

530 (Z201100005320016 to Y.H.), Beijing Advanced Innovation Center for Genomics,

531 and Shenzhen Bay Laboratory.

\section{Author Contributions}

534 Y.H., J.W., and Z.Z. conceived the study; L.L., H.C., J.Z., L.D., J.S., and S.G.

535 performed experiments; H.C., L.L. and M.D. performed data analyses; Y.F., L.L, L.D., 536 and H.C. developed the Tasc-WGS protocol; C.S., J.W., and Y.L provided clinical

537 samples; L.L., H.C., Y.H., and J.W. wrote the manuscript with inputs from all authors;

538 Y.H., J.W., and Z.Z. supervised all aspects of this study.

539

540 Competing Interests

541 Authors declare no competing interests.

\section{References}

544 Amos-Landgraf JM, Cottle A, Plenge RM, Friez M, Schwartz CE, Longshore J,

545 Willard HF. 2006. X Chromosome-inactivation patterns of 1,005

546 phenotypically unaffected females. The American Journal of Human Genetics

547 79: 493-499.

548 Beroukhim R, Mermel CH, Porter D, Wei G, Raychaudhuri S, Donovan J, Barretina

549 J, Boehm JS, Dobson J, Urashima M et al. 2010. The landscape of somatic

$550 \quad$ copy-number alteration across human cancers. Nature 463: 899-905. 
551 Bundo M, Toyoshima M, Okada Y, Akamatsu W, Ueda J, Nemoto-Miyauchi T,

552 Sunaga F, Toritsuka M, Ikawa D, Kakita A et al. 2014. Increased L1 retrotransposition in the neuronal genome in schizophrenia. Neuron 81: 306313.

Cai X, Evrony GD, Lehmann HS, Elhosary PC, Mehta BK, Poduri A, Walsh CA. 2014. Single-cell, genome-wide sequencing identifies clonal somatic copynumber variation in the human brain. Cell reports 8:1280-1289.

Chen C, Xing D, Tan L, Li H, Zhou G, Huang L, Xie XS. 2017. Single-cell wholegenome analyses by Linear Amplification via Transposon Insertion (LIANTI). Science 356: 189-194.

Chronister WD, Burbulis IE, Wierman MB, Wolpert MJ, Haakenson MF, Smith AC, with complex karyotypes are rare in aged human neocortex. Cell reports. 26: $825-835$.

Conrad DF, Pinto D, Redon R, Feuk L, Gokcumen O, Zhang Y, Aerts J, Andrews TD, number variation in the human genome. Nature 464: 704-712.

DePristo MA, Banks E, Poplin R, Garimella KV, Maguire JR, Hartl C, Philippakis

572 Erwin JA, Paquola AC, Singer T, Gallina I, Novotny M, Quayle C, Bedrosian TA, discovery and genotyping using next-generation DNA sequencing data. Nature genetics 43: 491-498.

576 Evrony GD, Lee E, Mehta BK, Benjamini Y, Johnson RM, Cai X, Yang L, Haseley P, 577 Lehmann HS, Park PJ et al. 2015. Cell lineage analysis in human brain using 578 endogenous retroelements. Neuron 85: 49-59. 
579 Galupa R, Heard E. 2018. X-chromosome inactivation: a crossroads between

$580 \quad$ chromosome architecture and gene regulation. Annual review of genetics 52:

$581 \quad 535-566$.

582 Girirajan S, Campbell CD, Eichler EE. 2011. Human copy number variation and 583 complex genetic disease. Annual review of genetics 45: 203-226.

584 Hassold T, Hunt P. 2001. To err (meiotically) is human: the genesis of human aneuploidy. Nature Reviews Genetics 2: 280-291.

Jacobs KB, Yeager M, Zhou W, Wacholder S, Wang Z, Rodriguez-Santiago B, mosaicism and its relationship to aging and cancer. Nature genetics 44: 651658.

Klopocki E, Mundlos S. 2011. Copy-number variations, noncoding sequences, and human phenotypes. Annual review of genomics and human genetics 12:53-72.

Knouse KA, Wu J, Amon A. 2016. Assessment of megabase-scale somatic copy number variation using single-cell sequencing. Genome research 26: 376-384.

Knouse KA, Wu J, Whittaker CA, Amon A. 2014. Single cell sequencing reveals low levels of aneuploidy across mammalian tissues. Proceedings of the National Academy of Sciences 111: 13409-13414.

Laks E, McPherson A, Zahn H, Lai D, Steif A, Brimhall J, Biele J, Wang B, Masud T, Ting J et al. 2019. Clonal decomposition and DNA replication states defined by scaled single-cell genome sequencing. Cell 179: 1207-1221.

600 Langmead B, Trapnell C, Pop M, Salzberg SL. 2009. Ultrafast and memory-efficient 601 alignment of short DNA sequences to the human genome. Genome biology 10:

603 Laurie CC, Laurie CA, Rice K, Doheny KF, Zelnick LR, McHugh CP, Ling H, 604 Hetrick KN, Pugh EW, Amos C et al. 2012. Detectable clonal mosaicism from 605 birth to old age and its relationship to cancer. Nature genetics 44: 642-650. 
606 Li H, Handsaker B, Wysoker A, Fennell T, Ruan J, Homer N, Marth G, Abecasis G,

607 Durbin R. 2009. The sequence alignment/map format and SAMtools.

608 Bioinformatics 25: 2078-2079.

609 Machiela MJ, Zhou W, Karlins E, Sampson JN, Freedman ND, Yang Q, Hicks B, Dagnall C, Hautman C, Jacobs KB et al. 2016. Female chromosome X

Machiela MJ, Zhou W, Sampson JN, Dean MC, Jacobs KB, Black A, Brinton LA, Chang IS, Chen C, Chen C et al. 2015. Characterization of large structural genetic mosaicism in human autosomes. The American Journal of Human Genetics 96: 487-497. mosaicism is age-related and preferentially affects the inactivated $\mathrm{X}$ sequencing. Nature 472: 90-94.

623 Nilsen G, Liestøl K, Van Loo P, Vollan HK, Eide MB, Rueda OM, Chin SF, Russell R, Baumbusch LO, Caldas C et al. 2012. Copynumber: Efficient algorithms for single-and multi-track copy number segmentation. BMC genomics 13: 1-6.

Olshen AB, Venkatraman ES, Lucito R, Wigler M. 2004. Circular binary segmentation for the analysis of array-based DNA copy number data. Biostatistics 5: 557-572.

629 Richardson SR, Morell S, Faulkner GJ. 2014. L1 retrotransposons and somatic mosaicism in the brain. Annual review of genetics 48: 1-27.

631 Rohrback S, April C, Kaper F, Rivera RR, Liu CS, Siddoway B, Chun J. 2018.

632 Submegabase copy number variations arise during cerebral cortical 
neurogenesis as revealed by single-cell whole-genome sequencing. Proceedings of the National Academy of Sciences 115: 10804-10809.

Sanchez-Luque FJ, Kempen MJ, Gerdes P, Vargas-Landin DB, Richardson SR, Troskie RL, Jesuadian JS, Cheetham SW, Carreira PE, Salvador-Palomeque C et al. 2019. LINE-1 evasion of epigenetic repression in humans. Molecular cell 75: 590-604.

Sandovici I, Naumova AK, Leppert M, Linares Y, Sapienza C. 2004. A longitudinal study of X-inactivation ratio in human females. Human genetics 115: 387-392.

Santaguida S, Amon A. 2015. Short-and long-term effects of chromosome missegregation and aneuploidy. Nature reviews Molecular cell biology 16: 473485 .

Shah SP, Xuan X, DeLeeuw RJ, Khojasteh M, Lam WL, Ng R, Murphy KP. 2006. Integrating copy number polymorphisms into array $\mathrm{CGH}$ analysis using a robust HMM. Bioinformatics 22: e431-e439.

Sharp A, Robinson D, Jacobs P. 2000. Age-and tissue-specific variation of X chromosome inactivation ratios in normal women. Human genetics 107: 343349.

Sudmant PH, Rausch T, Gardner EJ, Handsaker RE, Abyzov A, Huddleston J, Zhang Y, Ye K, Jun G, Fritz MH et al. 2015. An integrated map of structural variation in 2,504 human genomes. Nature 526: 75-81.

The Wellcome Trust Case Control Consortium. 2010. Genome-wide association study of CNVs in 16,000 cases of eight common diseases and 3,000 shared controls. Nature 464: 713-720.

van den Bos H, Spierings DC, Taudt A, Bakker B, Porubský D, Falconer E, Novoa C, Halsema N, Kazemier HG, Hoekstra-Wakker K et al. 2016. Single-cell whole genome sequencing reveals no evidence for common aneuploidy in normal and Alzheimer's disease neurons. Genome biology 17: 1-9. 
660 Vattathil S, Scheet P. 2016. Extensive hidden genomic mosaicism revealed in normal tissue. The American Journal of Human Genetics 98: 571-578.

Vitak SA, Torkenczy KA, Rosenkrantz JL, Fields AJ, Christiansen L, Wong MH, genomes with combinatorial indexing. Nature methods 14: 302-308.

Waddell N, Pajic M, Patch AM, Chang DK, Kassahn KS, Bailey P, Johns AL, Miller D, Nones K, Quek K et al. 2015. Whole genomes redefine the mutational landscape of pancreatic cancer. Nature 518: 495-501.

Yurov YB, Iourov IY, Vorsanova SG, Liehr T, Kolotii AD, Kutsev SI, Pellestor F, Beresheva AK, Demidova IA, Kravets VS et al. 2007. Aneuploidy and confined chromosomal mosaicism in the developing human brain. PloS one 2: e558.

Zahn H, Steif A, Laks E, Eirew P, VanInsberghe M, Shah SP, Aparicio S, Hansen CL. 2017. Scalable whole-genome single-cell library preparation without preamplification. Nature methods 14: 167-173.

Zarrei M, MacDonald JR, Merico D, Scherer SW. 2015. A copy number variation map of the human genome. Nature reviews genetics 16: 172-183. single-cell sequencing. Nature communications 6: 1-10. health, disease, and evolution. Annual review of genomics and human genetics 10: $451-481$. 
bioRxiv preprint doi: https://doi. org/10.1101/2021.11.10.468149; this version posted November 13, 2021. The copyright holder for this
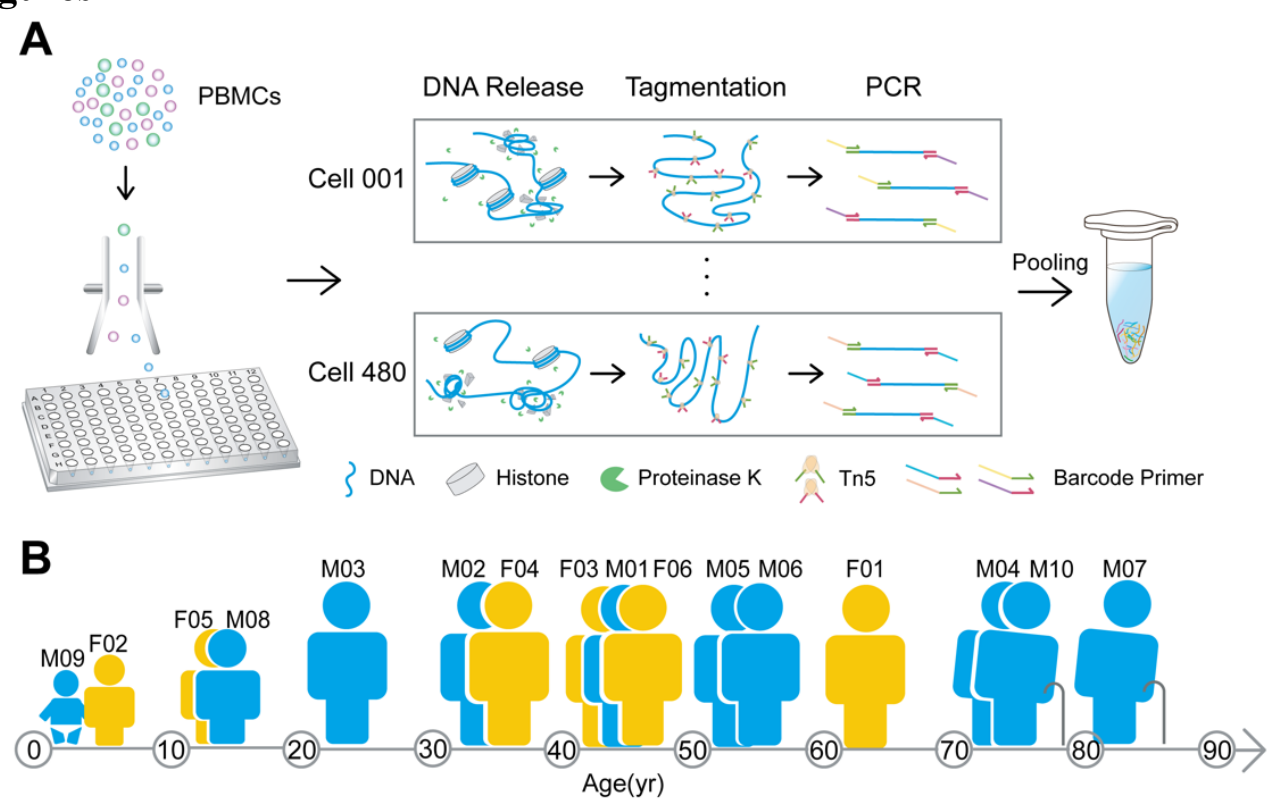

C Sequencing depth
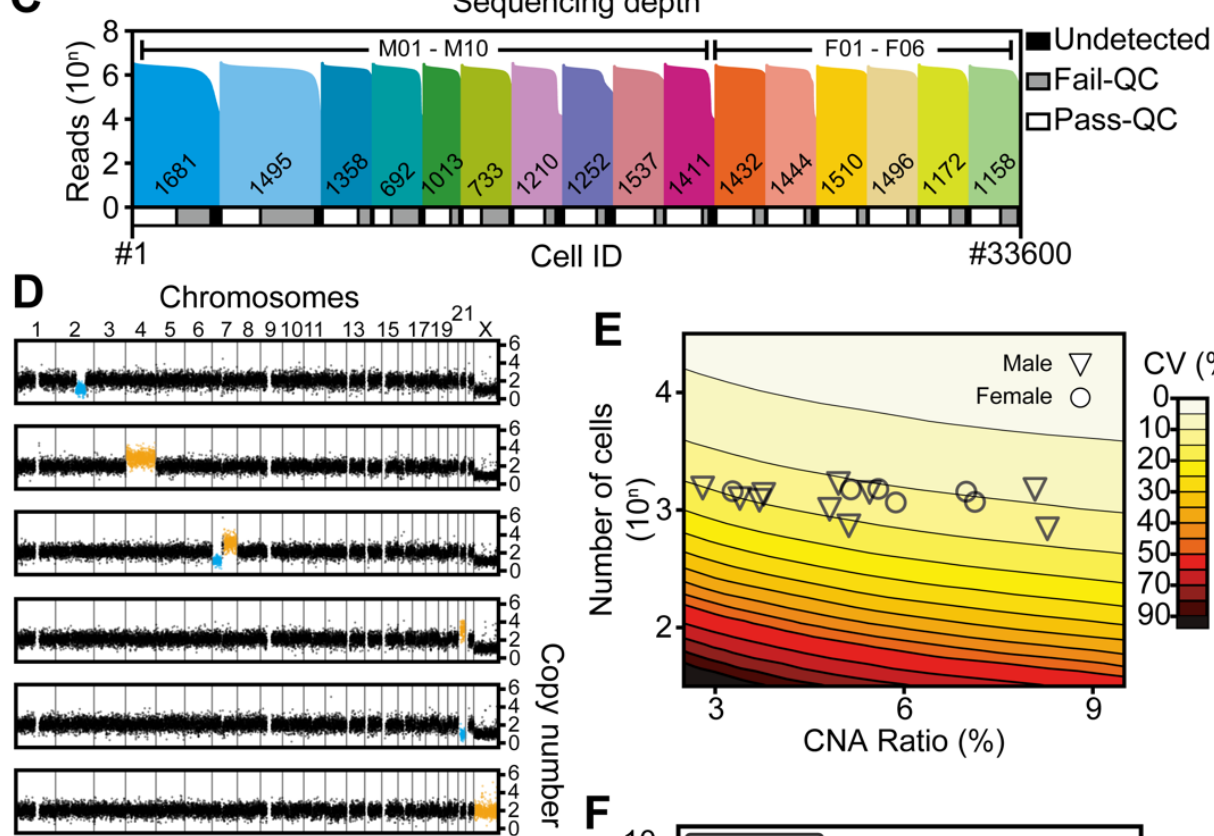

Cell ID \#33600

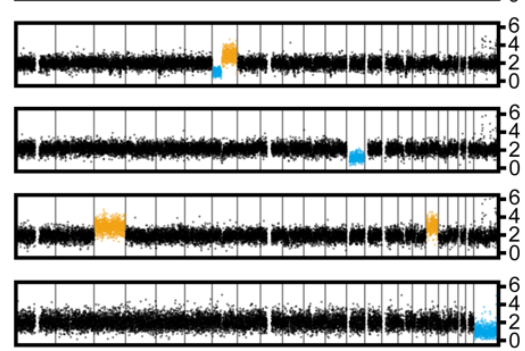

Copy number alterations $-2 \quad-1 \quad 0+1+2$
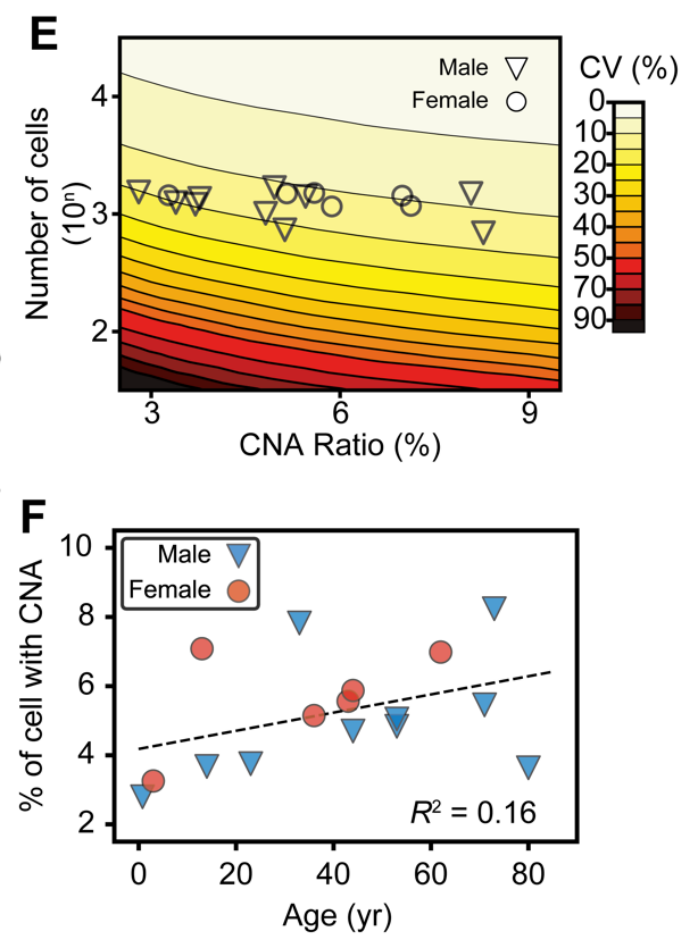

689 Fig. 1. Overview of the study design. (A) Experimental flow used in this study.

690 Lymphocytes were sorted to 96-well plates using fluorescence-activated cell sorting

691 to obtain 1 cell per well. Thousands of those single-cells were lysed, tagmented,

692 barcoded, and amplified in their wells and then pooled for second-generation 
693 sequencing. PBMC, peripheral blood mononuclear cells. (B) Cartoon showing the

694 blood donors' ages, sex, and ID numbers: 6 females (F) and 10 males (M) between 9

695 months and 80 years of age. (C) Sequencing depths and cell numbers for all samples

696 in this study. The top histogram shows the reads counts distribution and the diagram

697 underneath represents the relative proportions of cells after filtering. Undetected, low

698 read counts; Fail-QC, failed quality filtering; Pass-QC, passed quality filtering. (D)

699 Copy number profiles of representative cells with copy number alterations (CNAs) in

700 colors. (E) Coefficients of variation (CV) of CNA ratio estimations. The contour plot

701 shows both the theoretical CVs of CNA ratios (calculated by simulation) and the

702 sample sizes (number of cells). The symbols show the real CNA ratios and sample

703 sizes for each sample in this study. The large sample size in our study ensured that the

704 CV was in a relatively small interval, thus providing acceptably accurate estimations.

705 (F) Ages and autosomal CNA percentages of each sample. The dashed line indicates a 706 weak linear relationship between age and CNA ratios. 
A
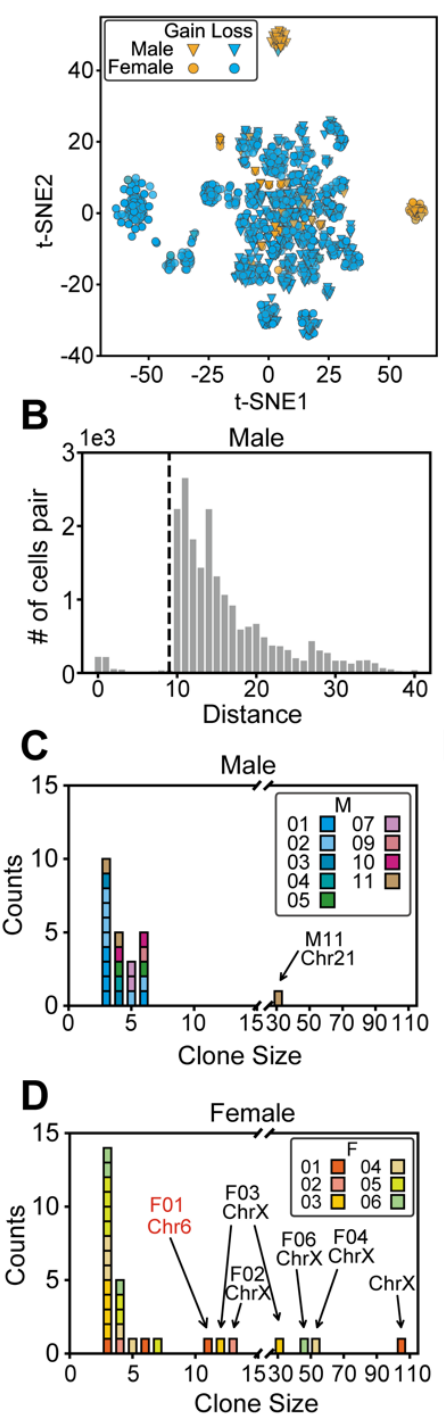
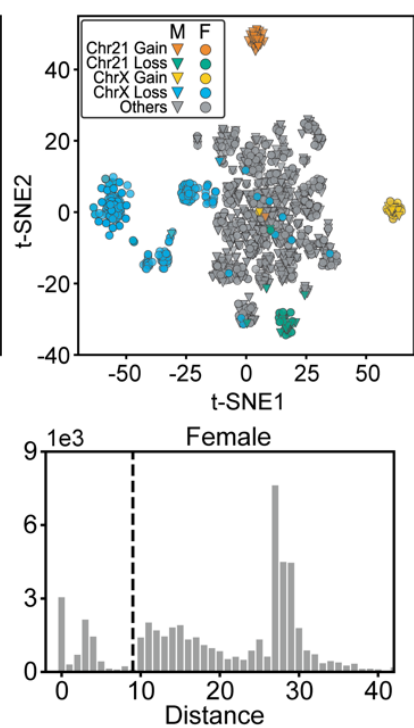

E F01 Chr6 clonal CNA cells
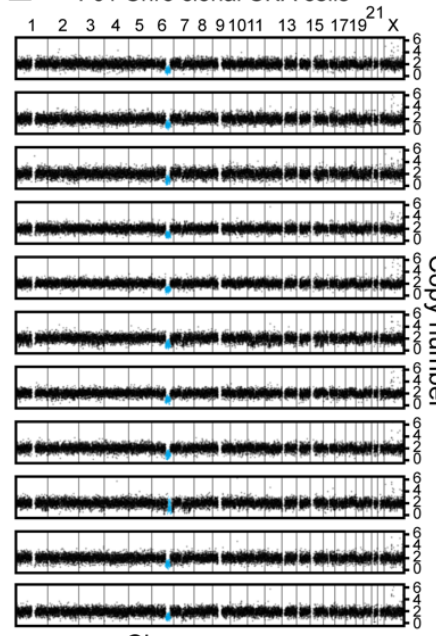

Chromosomes
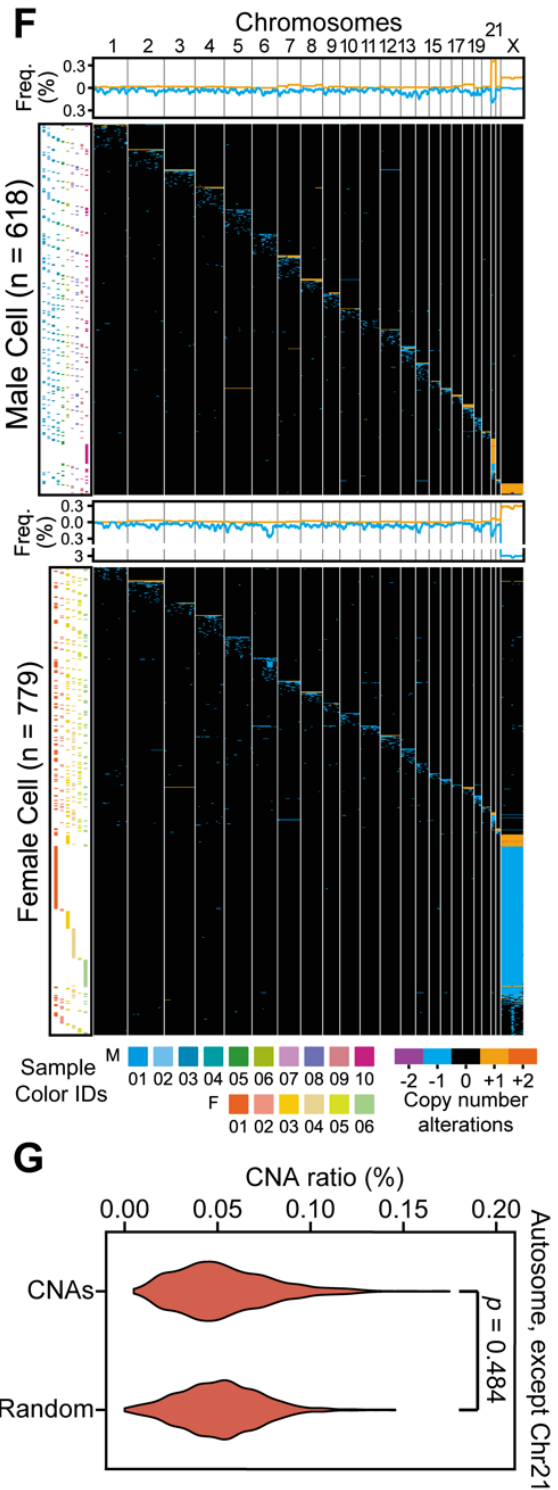

Fig. 2. The landscape of cells with copy number alterations (CNAs). (A) Low-

710 dimensional representations produced through multidimensional scaling of the copy

711 number profiles for cells with $>10-\mathrm{Mb}$ CNAs. Colors label different types and

712 locations of CNAs. t-SNE, $t$-distributed stochastic neighbor embedding. (B) The

713 distributions of Euclidean distances between cell pairs. Two clusters were clearly

714 separated by distance $(d)<10$ (indicated by dashed lines) in both male and female

715 samples. (C, D) The sizes (number of cells in a clone) and counts of clonal CNAs.

716 Each block in the bar plot represents a clone. Most clonal CNAs with bigger clone

717 sizes were on chr 21 and chr X, although there are some small clones with cell

718 numbers of about 3-5. (E) Copy number profiles of each clonal CNA on chr 6 in 11

719 cells from F01. Each graph represents 1 cell and the blue dots represent the regions

720 with copy number alterations. (F) Overview showing every cell with $>10-\mathrm{Mb}$ CNAs

721 each. The heatmap (in black) demonstrates the genome patterns for cells with CNAs,

722 which are labeled by different colors for gain and loss. Cells were sorted according to

723 the chromosome fraction carrying CNAs. Each row represents one cell. The scatter 
724 plot (left) shows the cells' sample IDs. The density curve along the top of each

725 heatmap shows the aggregate frequency, at 200-kb bins, among all samples for each

726 genomic locus. (G) CNA frequency distribution for each genomic locus in all

727 autosomes except for Chr 21. The distributions of this study's CNAs and of randomly

728 generated CNAs were not significantly different (Mann-Whitney $U$ test), thus

729 indicating a random generation mechanism.

730 
A

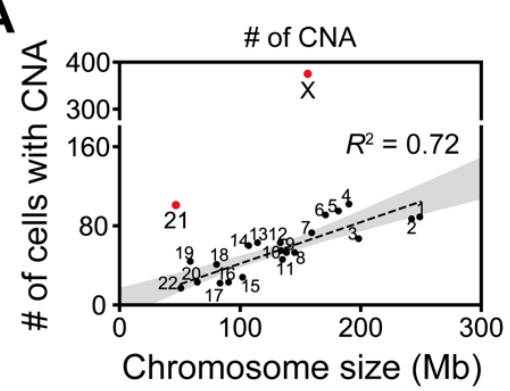

C

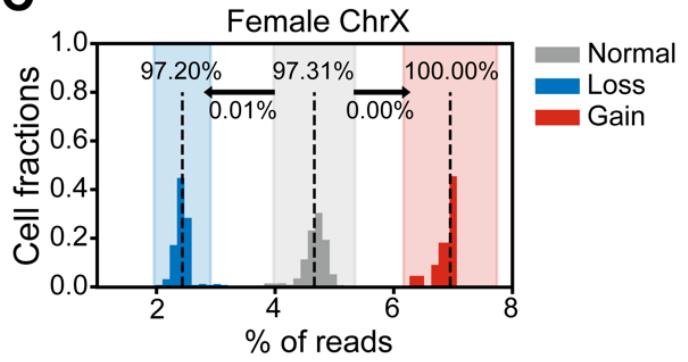

B $\quad$ Chr1

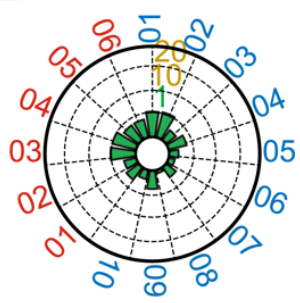

Chr8

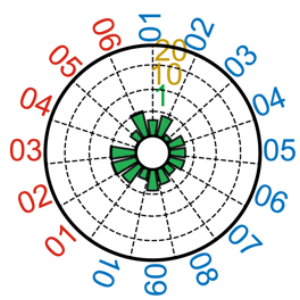

Chr21

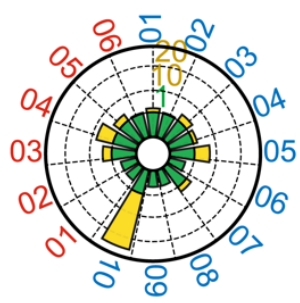

ChrX

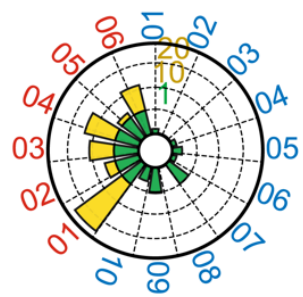

D $1.0 \quad \mathrm{Chr} 21$

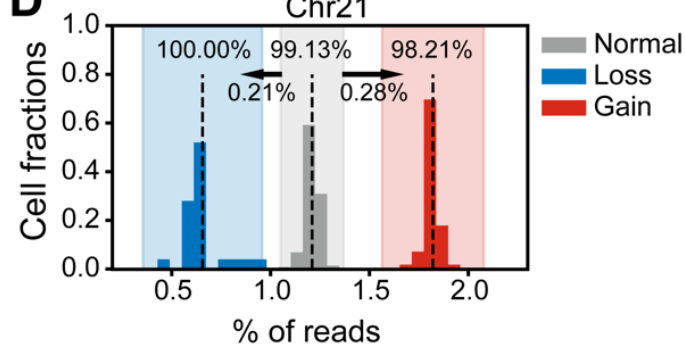

$\mathbf{E}$
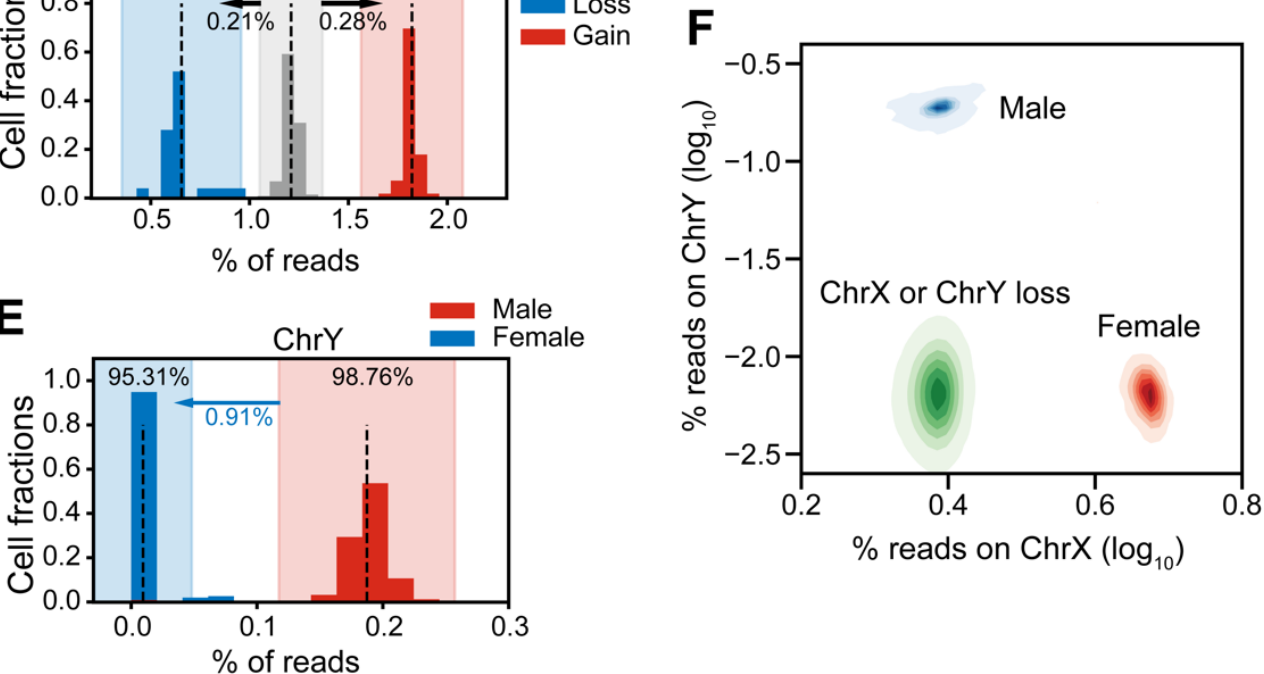

Fig. 3. Clonal analysis of cells with CNAs. (A) Numbers of cells with CNAs for each chromosome. The dashed line indicates a significant correlation between chromosome size and numbers of CNAs. Chromosomes 21 and X (red) had markedly higher numbers of CNAs than the rest of the chromosomes had. (B) Radar plots show normalized CNA counts in 4 chromosomes from every individual labeled around each plot (blue, male; red, female). Internal colors denote a graded scale of CNA counts (green, 0-1; yellow, 1-20). (C-E) Reads counts distributions on Chromosomes X (C), 21 (D), and Y (E). Each histogram shows the normalized reads distributions for each type of cells (gain, loss, and normal). Dashed lines indicate the means of each type of cell. The color shade indicates the confidence interval within 3 standard deviations. The percentage in each shaded bar indicates the fraction of cells identified as normal/loss/gain using reads counts. The percentages under the arrows shows the

744 fractions of normal cells found, using reads counts, in the loss/gain confidence 745 intervals. For Chromosome Y, those male cells that fell into the female confidence 
746 interval were identified as Chromosome Y losses. (F) Reads densities of Chr X and 747 Chr Y of normal male cells (blue), normal female cells (red), and abnormal male and 748 female cells with Chr Y or Chr X loss, respectively (both green). 


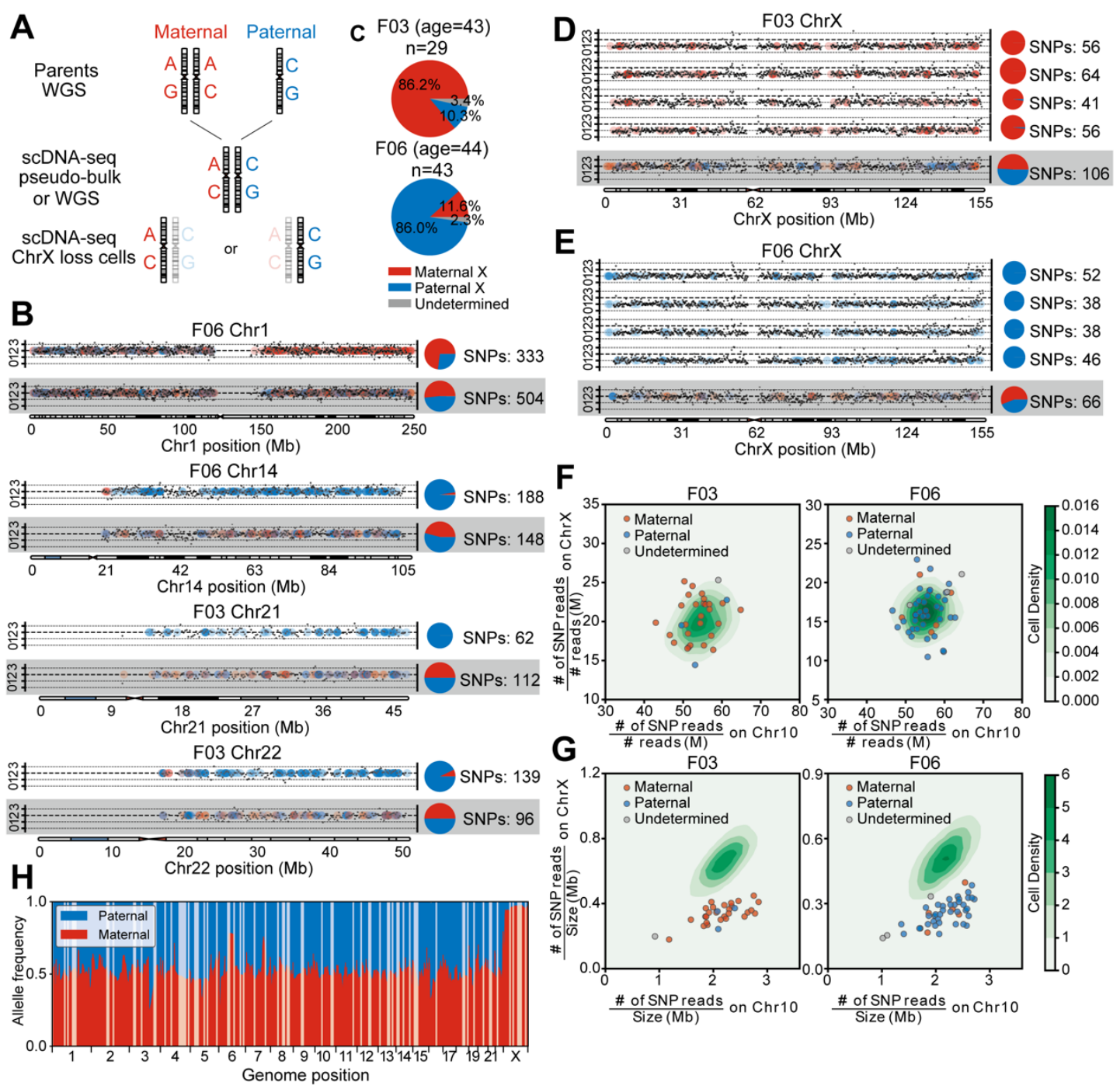

Fig. 4. Single-cell (female) haplotype analysis. (A) Haplotype identification pipeline of representative chromosome loss cells. For each candidate, two parental genomes

753 were sequenced by whole genome sequencing (WGS). Then, using WGS data or 754 merged single-cell data (pseudo-bulk), we identified each candidate's heterogeneous 755 sites. Combined with parental data, the heterogeneous sites were labeled paternal or 756 maternal. Finally, we analyzed each candidate's single cells that had chromosome loss 757 and extracted heterogeneous sites covered by reads, identifying each as paternal or 758 maternal. (B) Copy number profiles and haplotype identifications of cells with lost 759 heterozygosity. Black dots show the copy number for each genome locus and dashed 760 lines indicate the integer copy number $(0,1,2,3)$. Colored dots represent the sources of 761 a heterozygous site (red, maternal; blue, paternal) and the pie charts demonstrate the 762 paternal/maternal compositions (numbers of single-nucleotide polymorphisms 763 [SNPs]) of heterozygous sites on each chromosome. Results from normal cells are 764 shown on the bottom for comparison. (C) Paternal and maternal allele composition of 765 two individuals' cells with lost X Chromosomes. Most of the X-loss cells were of the 766 same parental allele. (D, E) Copy number profiles and haplotype identifications of 767 cells with lost X Chromosomes and of normal cells, as controls (bottom lines). (F) 
768 SNP densities and (G) reads densities on chromosomes of normal and ChrX-loss

769 cells. The contour plots show the normal cells' distributions and the scatter plots show

770 the ChrX-loss cells. Cells with lost X Chromosomes that have lower reads density on

771 ChrX, but comparable SNPs density, were not contaminated by male or parental cells.

772 (H) Allele compositions of heterogeneous sites in bulk RNA-seq reads of F03 cells.

773 Most of the genomic region displays bi-allelic expression (allele composition $\sim 50 \%$ ),

774 but Chr X clearly shows a maternal bias, which is corrected when most Chr X-loss

775 cells are paternal.

776 